\title{
Thyroid Lid Surgery
}

\author{
V. T. THALLER, K. KADEN, C. M. LANE and J. R. O. COLLIN \\ London
}

\begin{abstract}
Summary
A retrospective study of 58 thyroid patients undergoing eyelid surgery for thyroid related lid malposition is reported. A treatment strategy is suggested, based on the results of this experience.

The following points are stressed:

(1) The importance of recognising and relieving the inferior rectus tethering component of upper lid retraction when present.

(2) The usefulness of a scleral graft in lower lid retractor recession.

(3) The inadequacy of lateral tarsorrhaphy in relieving lid retraction but its value in camouflage.
\end{abstract}

Thyroid ophthalmopathy affects the extraocular muscles with an inflammatory swelling which leads to eventual fibrosis. The effects are aggravated by deposition of mucopolysaccharides in the lid and orbital connective tissue and may result in optic nerve compression, proptosis and lid retraction with ocular motility restriction, tear film disorder, corneal exposure and lid margin malposition.

Optic nerve compression or sight threatening corneal exposure require urgent medical or surgical orbital decompression (rarely central tarsorrhaphy). Although this treatment usually reduces proptosis, lid retraction may remain unaltered or in some cases, be worsened.

Diplopia is the next most disturbing symptom of thyroid eye disorder and extraocular muscle surgery is often required to maximise the field of binocular single vision around the primary position of gaze. This squint surgery may affect lid position.

Once the above problems have been adequately treated and the eye signs have become stable, patients often have persistent ocular exposure symptoms and the majority are cosmetically disfigured. It is at this stage of rehabilitation of the thyroid patient that eyelid surgery can play an important role (Fig. 1). ${ }^{1.2}$

We present our experience in the management of thyroid lid disorders since 1977 and on the basis of our results, propose a treatment plan for the logical selection of appropriate operations from the many that have been described. We do not pretend that ours is the only correct approach but feel that it is supported by our results.

\section{Material and Methods}

This is a retrospective study of 58 consecutive patients treated for thyroid lid disease at the Oculoplastic Service at Moorfields Eye Hospital since 1977. The age and sex ratio are presented in Table I and although this series comprises second-

From: Oculoplastic Unit, Moorfields Eye Hospital, London.

Correspondence to: Mr. J. R. O. Collin, Moorfields Eye Hospital, City Road, London EC1V 2PD.

Presented at the Oxford Ophthalmological Congress, July 1986. 
THYROID EYE MANAGEMENT STRATEGY

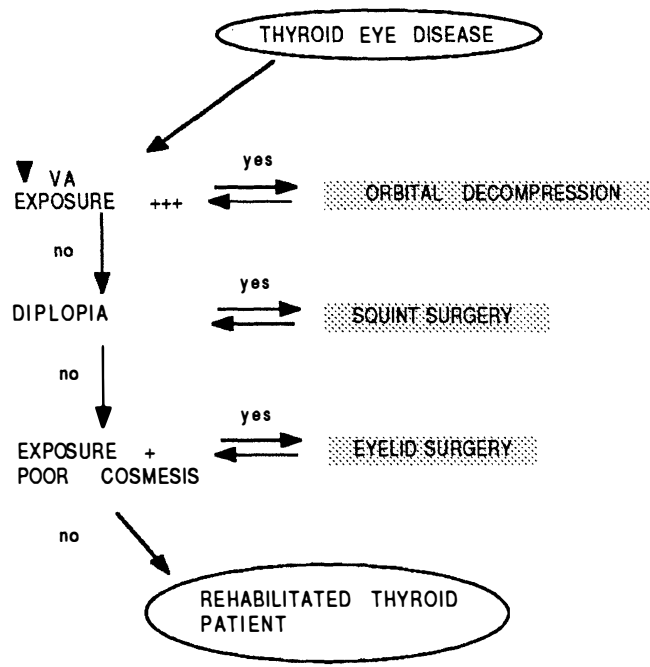

Fig. 1. Flow chart showing the position of eyelid surgery in the total management of thyroid eye disease.

ary and tertiary referrals, the age range and sex ratio are similar to that reported by Doxanas. ${ }^{3}$

Thirty of our patients had undergone previous thyroid related eye surgery, predominantly squint surgery and orbital decompression and seventeen had had lid surgery, lateral tarsorrhaphy being the most common procedure (two-thirds).

The diagnosis of thyroid disease had been established prior to the patients being referred to us on clinical and/or biochemical grounds.

All the patients in our series complained of unacceptable cosmesis as a result of their thyroid ophthalmopathy and two-thirds had persistent corneal exposure signs and symptoms in spite of the use of topical lubricants.

\section{Surgery}

The lid surgery was carried out under either local or general anaesthetic. Local anaesthesia was always preferred if the patient would tolerate it except in cases with inferior rectus recessions. The procedures fell into four main groups:

(1) Inferior rectus recession.

(2) Upper lid retractor recession \pm scleral graft.

(3) Lower lid retractor recession with scleral graft

(4) Cosmetic tarsorrhaphy and/or blepharoplasty.

\section{Inferior Rectus Recession}

Where inferior rectus tethering contributed significantly to upper lid retraction as evidenced by increased upper lid retraction from downgaze to upgaze, the inferior rectus muscle insertion was recessed by $4 \mathrm{~mm}$. A special effort was made to separate the lower lid retractors from the inferior rectus sheath at the time of surgery and the lower lid placed on traction for 48 hours to avoid the complication of increased lower lid retraction.

\section{Upper Lid Retractor Recession}

The degree of upper lid retraction was graded as small, medium and large, as shown in Table II.

Small amounts of retraction $(<2 \mathrm{~mm})$ were treated with a Mullerotomy type procedure as described by Henderson. ${ }^{4}$

Moderate degrees of upper lid retraction underwent Mullerotomy with transection of the levator

Table I. Statistics of the group studied

\begin{tabular}{llcc}
\hline & & Female & Male \\
\hline No. patients & 58 & 47 & 11 \\
& & $(81 \%)$ & $(19 \%)$ \\
Average age & 43 yrs $(20-70)$ & & \\
Average follow-up & 20 months & & \\
Previous surgery & $52 \%$ & & \\
No prior surgery & $54 \%$ & \\
\hline
\end{tabular}

Table II. Degree of upper lid retraction in millimetres related to type of operation chosen.

\section{Levator Complex Recession}

\begin{tabular}{lrl}
\hline Small & $<2 \mathrm{~mm}$ & Muller's Recession \\
Medium & $2-3 \mathrm{~mm}$ & $\begin{array}{l}\text { Full Retractor Recession } \\
+/- \text { Lysis of Horns }\end{array}$ \\
Large & $>3 \mathrm{~mm}$ & $\begin{array}{l}\text { Retractor Recession } \\
\text { Lysis of Horns } \\
\text { Division of superior } \\
\text { suspensory ligament of fornix } \\
+/- \text { Scleral graft }\end{array}$ \\
\hline
\end{tabular}

Table III. Results of inferior rectus recession

\begin{tabular}{cccc}
\hline Patients & Good & Improved & Poor \\
\hline 4 & $75 \%$ & $25 \%$ & 0 \\
Good: & $\begin{array}{l}\text { More than 2 } \\
\text { retraction }\end{array}$ & mm lessening of upper lid \\
Improved: & $\begin{array}{l}1-2 \\
\text { mm lessening of upper lid retraction }\end{array}$ \\
Poor: & No change in upper lid position \\
\hline
\end{tabular}


Table IV. Results of upper lid retractor recession

\begin{tabular}{clccc}
\hline Patients & & Good & Improved & Poor \\
\hline 11 & Muller Recession & $27.5 \%$ & $45 \%$ & $27.5 \%$ \\
30 & Levator Recession & $62 \%$ & $27 \%$ & $11 \%$ \\
13 & Levator Recession and Sclera & $35 \%$ & $54 \%$ & $11 \%$ \\
5 & Z Myotomy & $20 \%$ & $80 \%$ & 0 \\
& Good: & Desired degree of upper lid rececssion with good lid margin contour & \\
& Improved: & Slightly under or over-corrected or poor contour & \\
& Poor: & Inadequate recession or gross over-correction & \\
\hline
\end{tabular}

Table V. Results of lower lid retractor recession with scleral graft

\begin{tabular}{lccc}
\hline \multicolumn{1}{c}{ Patients } & Good & Improved & Poor \\
\hline \multicolumn{1}{c}{25} & $82 \%$ & $14 \%$ & $4 \%$ \\
Good: & 2 or more mm elevation of lower lid \\
Improved: & $\begin{array}{l}\text { 1-2 mm elevation of lower lid } \\
\text { no change in lower lid height }\end{array}$ \\
\hline
\end{tabular}

aponeurosis via the same approach and at the same level. Orbital septum was then opened to release the preaponeurotic fat pad and thus confirm the complete separation of levator aponeurosis from its skin insertions.

Greater recession was obtained by also cutting the medial and lateral horns of the levator aponeurosis and where necessary, fibrous adhesions to Whitnall's ligament and/or Whitnall's ligament per se were divided. The lateral lysis was emphasised in cases with a marked lateral peak of the upper lid contour caused by perilacrimal fibrosis.

In cases of severe retraction, a preserved scleral graft $^{5}$ was sometimes inserted and covered with conjunctiva which had previously been dissected free of Muller's muscle and of the superior suspensory ligament of the fornix. The necessity for this additional procedure has been questioned by us and in the later cases in our series severe upper lid retraction was treated by careful and complete levator complex recession alone.
In 5 patients we endeavoured to preserve the level of the skin crease by performing an anterior approach levator ' $Z$ ' myotomy lid recession as described by Grove. ${ }^{6}$

\section{Lower Lid Retractor Recession}

When lower lid retraction of 2 or more millimetres was present, the lower lid retractors were recessed following the technique described by Doxanas ${ }^{3}$ via a conjunctival incision along the lower border of tarsal plate with the lower lid everted over a Desmarres retractor. A strip of Eye Bank sclera, stored in 70 per cent alcohol and washed in a minimum of three changes of sterile Ringer's solution for 24 hours preoperatively, was inserted, the vertical height being twice the desired eyelid correction. This graft was either left bare (a simpler operation) or covered with conjunctiva (which must be adequately freed from the inferior suspensory ligament of the fornix).

\section{Cosmetic Tarsorrhaphy and Blepharoplasty}

With lid retraction adequately relieved, a small permanent lateral tarsorrhaphy and occasionally a Lee $^{7}$ type medial canthoplasty was performed to camouflage residual proptosis, especially where it was asymmetrical.

Where the lids were excessively bulky, a two or four lid blepharoplasty was performed. For fat excision, the orbital septum was opened along its entire length to improve access in the already

Table VI. Results of tarsorrhaphy

\begin{tabular}{clccc}
\hline Patients & & Good & Improved & Poor \\
\hline 11 & Lateral Tarsorrhaphy & $73 \%$ & $27 \%$ & 0 \\
3 & Medial Canthoplasty & $33 \%$ & $67 \%$ & 0 \\
8 & Opening Tarsorrhaphy & $100 \%$ & 0 & 0 \\
& Good: $\quad$ implies adequate camouflage obtained & & \\
& Improved: implies further surgery might give more improvement & \\
& Poor: & not cosmetically improved & \\
& & &
\end{tabular}


Table VII. Results of 2 and 4 lid blepharoplasty

\begin{tabular}{cccc}
\hline Patients & Good & Improved & Poor \\
\hline 16 & $44 \%$ & $50 \%$ & $6 \%$
\end{tabular}

Good: implies patient satisfied with result

Improved: implies patient had hoped for greater improvement

Poor: (one patient with a residual localised fat prolapse in lower lid post-surgery)

restricted space between the proptosed globe and orbital rim.

\section{Results}

1. Inferior Rectus Recession (Table III)

Of the four patients who underwent this operation, three achieved significant reduction in

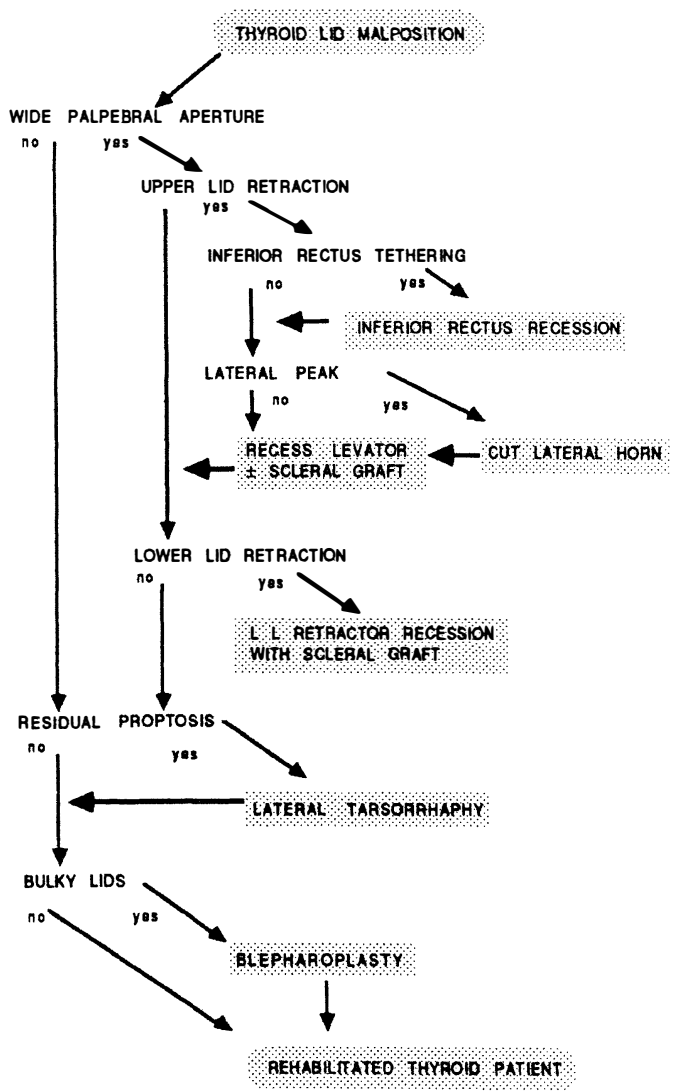

Fig. 2. Suggested scheme for selecting appropriate operations for the treatment of thyroid lid disorders. their upper lid retraction, the fourth showing only a slight improvement. The degree of subjective improvement was greater than shown by measurement in the primary position of gaze and all the patients were very much more comfortable. None of the patients developed diplopia as a result of this procedure. Indeed, their external ocular muscle balance was improved postoperatively as was their Bell's phenomenon.

2. Upper Lid Retractor Recession (Table IV)

(a) As a group, the Henderson type Mullerotomies were undercorrected over twothirds requiring further recession.

(b) Recession of the levator complex was performed in over half of our patients and had the best results with two-thirds requiring no further surgery. This group included moderate and severe upper lid retraction.

(c) Levator recession with scleral graft was again less predictable with only one-third requiring no further surgery. However, this was the worst affected group of patients.

(d) Anterior approach Z-myotomy in our hands, tended to undercorrect the patients and did not succeed in maintaining the upper lid skin crease at the desired level. Four of the five patients needed further correction.

In all fourteen patients had sufficient overcorrection to warrant subsequent ptosis surgery.

3. Lower Lid Retractor Recession (Table V) Of the 28 patients who underwent lower lid retractor recession with scleral graft, fourfifths achieved a minimum of $2 \mathrm{~mm}$ lower lid elevation, one-seventh only $1 \mathrm{~mm}$ and one patient had complete resorption of the graft with the lid position returning to its preoperative level.

\section{Tarsorrhaphy (Table VI)}

Lateral tarsorrhaphy for camouflage of proptosis in the absence of lid retraction was of considerable cosmetic help. Medial canthoplasty conferred only slight additional benefit.

Opening of primary, usually stretched, tar- 


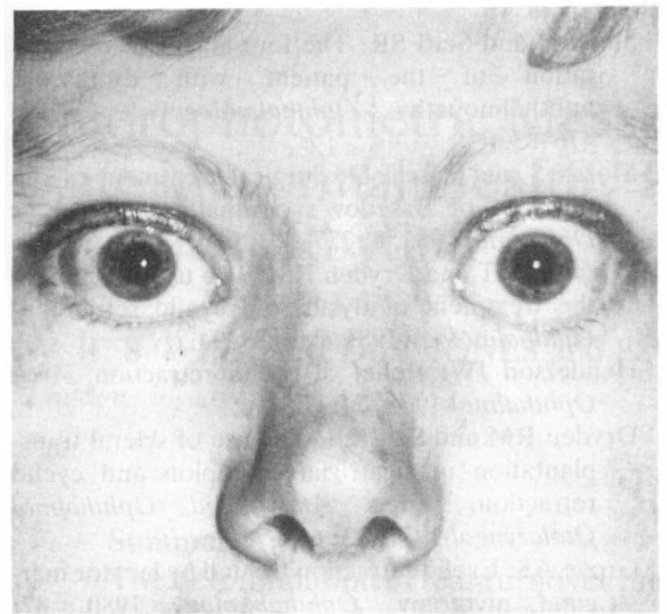

Fig. 3. A typical patient suffering from thyroid eye problems after bilateral surgical orbital decompression and lateral tarsorrhaphies.

sorrhaphies after successful relief of lid retraction was universally welcomed by the patients as a major improvement in their cosmesis.

\section{Blepharoplasty (Table VII)}

The majority of the patients were cosmetically improved by their blepharoplasties, but half had hoped for a greater improvement. These were primarily the ones with bulky upper lids. Lower lid blepharoplasty when performed, showed a more dramatic improvement.

\section{Discussion}

From the patients referred to us having undergone previous lid surgery, the most popular operation still appears to be primary lateral tarsorrhaphy. This is to be deplored as it cannot lessen lid retraction and is usually not able to withstand it either, becoming stretched and unsightly in the process. Henderson ${ }^{2}$ noted the deficiency of lateral tarsorrhaphy in 1965 and this prompted him to develop his Mullerotomy operation. Our result confirms Henderson's assertion that no patients are overcorrected by this procedure but show that the majority remain undercorrected, a fact which the photographs in Henderson's paper unwittingly demonstrate.

Combined posterior approach Mullerotomy and levator recession is a simpler procedure than Mullerectomy as described by Putterman $^{8}$ as Muller's muscle and con-

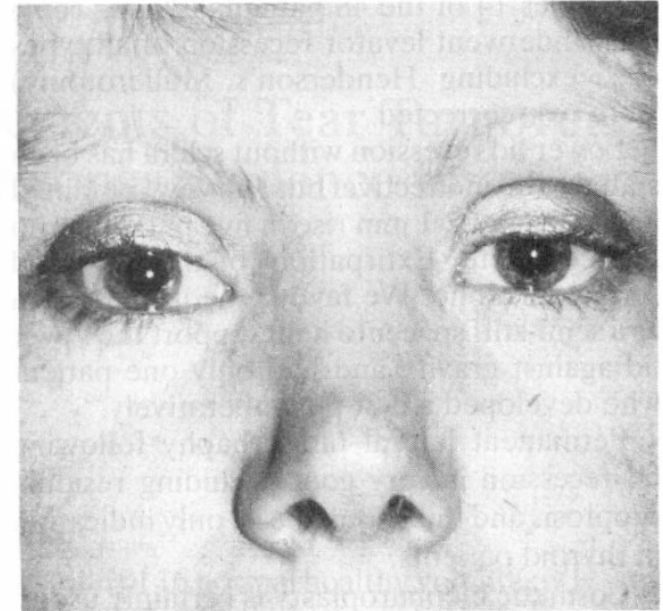

Fig. 4. Same patient as in Figure 3 after undergoing bilateral upper lid levator recessions and bilateral lower lid retractor recessions with scleral grafts.

junctiva are often fibrosed and difficult to dissect. In our hands, the former operation gave good results.

The primary indications for levator ' $Z$ ' myotomy given by Grove were 'division of skin adhesions' 6 and to control the height of the skin crease. ${ }^{9}$ The former is simple to achieve via the conjunctival route once the orbital septum is opened and we were singularly unsuccessful in achieving the latter by Grove's method.

We are tending to use sclera less often in the upper lid as our experience has shown that thorough lysis of the levator aponeurosis horns and any high support from Whitnall's ligament plus release of the superior suspensory ligament of the fornix can provide an ample amount of recession in most instances.

Putterman ${ }^{10}$ reiterated that increased levator tone is associated with inferior rectus tethering, which previous authors had noted, but he made no attempt to relieve the primary cause. We feel that where there is a demonstrable component of upper lid retraction resulting from inferior rectus tethering, the inferior rectus must be recessed initially or simple levator recessions will be doomed to failure.

The patient should be warned of the significant incidence of overcorrection following levator recession and that ptosis surgery might be required to deal with this complication. In 
our series 14 of the 48 patients ( 29 per cent) who underwent levator recession of all types (i.e. excluding Henderson's Mullerotomy) were overcorrected.

Lower lid recession without sclera has been stated to be ineffective ${ }^{4}$ but Harvey ${ }^{11}$ obtained an average of $2.1 \mathrm{~mm}$ rise in five patients who had complete 'Extirpation' of the lower lid retractors alone. We favour the use of sclera as a semi-stiff spacer to help support the lower lid against gravity and had only one patient who developed a cyst post-operatively.

Permanent lateral tarsorrhaphy following lid recession is very good at hiding residual proptosis and this should be its only indication in thyroid patients.

Cosmetic blepharoplasty is certainly useful in diminishing bulky lids but is more effective in the lower lids and often disappointing in the upper lids as there is seldom as much excess skin and fat in that area, the bulkiness being due to increased intra-orbital contents and proptosis.

On the basis of our results, we propose the flow chart (Fig. 2) as a logical approach to the symptomatic and cosmetic treatment of patients with thyroid lid disease. It requires multiple operations but is certainly worthwhile from the patient's point of view (Figs 3 and 4).

\section{References}

'Shorr N and Seiff SR: The four stages of rehabilitation of the patient with dysthyroid ophthalmopathy. Ophthalmology 1986, 93: 476-83.

${ }^{2}$ Morax S and Pascal D: Surgical treatment of the sequelae of Basedow's ophthalmopathy. $J \mathrm{Fr}$ Ophtalmol 1985, 8: 227-37.

${ }^{3}$ Doxanas MT and Dryden RM: The use of sclera in the treatment of dysthyroid eyelid retraction. Ophthalmology 1981, 88: 887-94.

${ }^{4}$ Henderson JW: Relief of eyelid retraction. Arch Ophthalmol 1965, 74: 205-16.

${ }^{5}$ Dryden RM and Soll DB: The use of scleral transplantation in cicatricial entropion and eyelid retraction. Trans Am Acad Ophthalmol Otolaryngol 1977, 83: 669-78.

${ }^{6}$ Grove AS: Eyelid retraction treated by levator marginal myotomy. Ophthalmology 1980, 87: 1013-18.

${ }^{7}$ Lee OS: Operation for correction of everted lacrimal puncta. Am J Ophthalmol 1951, 34: 575.

${ }^{8}$ Putterman A and Urist M: Surgical treatment of upper lid retraction. Arch Ophthalmol 1972, 87: 401-5.

9 Grove AS: Upper eyelid retraction and Graves' disease. Ophthalmology 1981, 88: 499-506.

${ }^{10}$ Putterman AM: Surgical treatment of thyroid related upper eyelid retraction: Graded Müller's muscle excision and levator recession. Ophthalmology 1981, 88: 507-12.

"Harvey JT and Anderson RL: The aponeurotic approach to eyelid retraction. Ophthalmology 1981, 88: 513-24. 